\title{
A simple posturographic method detects the difference in recovery of patients receiving endoscopic sedation with propofol vs. midazolam
}

\author{
Mandel $\mathrm{JE}^{\mathrm{I}^{*}}$, Tietäväinen $\mathrm{A}^{2}$, Otero $\mathrm{J}^{3}$ and Hæggström $\mathrm{E}^{2}$ \\ ${ }^{1}$ Department of Anesthesiology \& Critical Care, Perelman School of Medicine, University of Pennsylvania, USA \\ ${ }^{2}$ Department of Physics, University of Helsinki, Finland \\ ${ }^{3}$ Robert Wood Johnson Medical School, USA
}

\begin{abstract}
Background: Measurement of the residual effects of sedatives on fitness for ambulation is typically performed with subjective measures, if at all. Previous efforts to objectively measure these effects employed dynamic posturography with expensive equipment that is not easily brought to the bedside and carries a risk of patient injury. A simple test employing consumer off-the-shelf technology that exposes the patient to no more risk than standing still might permit a more reliable assessment of fitness for discharge. We have previously shown that a simple tester, using a Nintendo Wii ${ }^{\circledR}$ Balance board and software that determined fuzzy sample entropy of postural sway can separate outpatients' postural steadiness before (PRE) and after (POST) endoscopy with midazolam/fentanyl conscious sedation. Propofol/fentanyl monitored anesthesia care also alters balance, but recovery is known to be more rapid. We hypothesized that the ability to detect decrements in postural steadiness with fuzzy sample entropy following monitored anesthesia care would be less than that seen in conscious sedation.
\end{abstract}

Methods: Fuzzy sample entropy of sway was measured in 92 patients undergoing monitored anesthesia care and 103 patients undergoing conscious sedation for minor endoscopic procedures under conditions of eyes open and closed in the PRE and POST states. The ability to discriminate the PRE and POST states was assessed by the area under the receiver operating characteristic curve (AUC).

Results: In patients undergoing conscious sedation, fuzzy sample entropy was lower both with eyes open and closed (0.83 and 0.80$)$. Following monitored anesthesia care, the PRE and POST states were less separable, with AUC of 0.56 and 0.60 (eyes open and closed).

Conclusions: A simple tester that determines fuzzy sample entropy of postural sway yields a result that is expected when applied to groups receiving different sedation modalities. The utility of this tester in assessing outcomes will require further evaluation.

\section{Introduction}

Patients undergoing elective endoscopy frequently require sedation. While sedation improves patient acceptance of the procedure, and may improve procedural outcome, sedation is associated with a period of impaired postural steadiness. Accurate assessment of the degree of decreased postural steadiness can be challenging, and measures such as time to recovery room discharge or ambulation without assistance may be subject to bias and inter-observer variability. An objective method of assessment could address these limitations.

Posturography measures the trajectory of the ground projection of the center-of-pressure of a subject standing on an instrumented surface. Posturography may be static or dynamic, that is, employ exogenous stimuli to introduce a disturbance into posture, such as a moving platform or a shifting artificial horizon. Previous studies of sedation effects on postural stability focused on computerized dynamic posturography as it has greater sensitivity than static posturography. Fujisawa demonstrated that computerized dynamic posturography was affected by midazolam [1], that these effects persisted longer in the elderly than in young people [2], and that the effects were less persistent with propofol sedation [3]. These authors further demonstrated that the results of the timed up and go test, in which a patient stood up, walked five meters, turned, and returned to their chair, correlated with the computerized dynamic posturography score [3,4]. Although computerized dynamic posturography and the timed up and go test may be sensitive measures of recovery, these tests may not be appropriate for all patients. Patients standing on a moving platform or walking and turning may suffer injuries as severe as those we seek to avoid. Thus, static posturography may be preferable to dynamic posturography when assessing patients recovering from anesthesia or sedation.

Posturography typically employs simple measures of postural sway such as velocity and sway radius. Since robust control of posture requires random perturbation of the center of pressure to permit dynamic tuning of the system [5], measures that assess entropy may more efficiently identify a decrement in capacity to maintain upright stance than the simpler measures [6,7]. We previously demonstrated the superiority of fuzzy sample entropy over other linear and nonlinear

Correspondence to: Mandel JE, Department of Anesthesiology \& Critical Care, Perelman School of Medicine, University of Pennsylvania, 3400 Spruce Street, Philadelphia, PA 19104, USA, E-mail: MandelJ@uphs.upenn.edu/Jeff. Mandel@uphs.upenn.edu

Received: March 14, 2017; Accepted: April 14, 2017; Published: April 17, 2017 
measures of postural sway in differentiation of the pre-sedative state from the post-recovery state in patients undergoing midazolamfentanyl based conscious sedation [8]. We have also demonstrated that time to ambulation without assistance was shorter with propofolbased sedation than with midazolam-based sedation [9] indeed, in the propofol group time to ambulation was less than the minimum time that patients are required to stay in the recovery room prior to discharge, while in the midazolam group, it was almost identical to this time. It is therefore likely that patients receiving monitored anesthesia care are more completely recovered at discharge than patients receiving conscious sedation. We hypothesized that this difference in recovery to street readiness between midazolam and propofol-based sedation would be demonstrable with fuzzy sample entropy of postural sway.

\section{Methods}

The study was approved by the Institutional Review Board of the University of Pennsylvania, School of Medicine, and was deemed exempt from written consent. All patients gave verbal consent for participation. Patients were recruited from the elective ambulatory practice of the Penn Endoscopy Center. Patients were scheduled for conscious sedation or monitored anesthesia care by their attending gastroenterologists. Patients were not randomized to treatment groups, and the only exclusion criteria were preexisting balance disorder or inability to ambulate unaided prior to the procedure. Convenience samples of 103 patients undergoing conscious sedation and 92 patients undergoing monitored anesthesia care were studied. In the group utilizing conscious sedation, the gastroenterologist directed a nurse to administer midazolam and fentanyl at intervals of 2-5 minutes until the patient was judged adequately sedated. In the group undergoing monitored anesthesia care, an anesthesia provider administered propofol to maintain deep sedation. Gastroenterologists and anesthesia providers were not informed that patients were enrolled in the study, and employed their usual practices. The 103 patients in conscious sedation were utilized in our previous effort [8], which identified fuzzy sample entropy as the most useful method for measuring the effects of sedation on postural steadiness.

Patients were evaluated prior to entering the endoscopy suite (PRE) and after meeting recovery room discharge criteria (POST). Patients were instructed to stand on a Nintendo Wii Balance Board, keep their hands at their sides, their feet in a comfortable stance and to fix their eyes on one spot (eyes open) or to close their eyes (eyes closed). An investigator stood next to the patient at all times ready to prevent the patient from falling.

Data was acquired from the board over the Bluetooth wireless data stream on a laptop computer running custom software written in Labview (National Instruments, Austin, TX) that recorded data at $60 \mathrm{~Hz}$ sampling frequency for subsequent analysis. Center-of-pressure sequences for anterior-posterior sway were utilized, as previous efforts had failed to demonstrate significant findings in lateral sway. The data sequences underwent initial nonlinear filtering to exclude artifacts and remove low frequency components. These sequences were then analyzed with fuzzy sample entropy using parameters $\mathrm{m}=3, \mathrm{r}=0.05$ and $c=0.01$, as we have previously described [8].

Comparisons of patients' age, height, and weight between conscious sedation and monitored anesthesia care groups were estimated with independent student's t-tests. Comparison of sex ratios was by Pearson's Chi-Square. All fuzzy sample entropy data was logtransformed before statistical analysis. A mixed-design ANOVA, with conscious sedation/monitored anesthesia care as between-subject factor, eyes open/eyes closed and PRE/POST as within-subject factors, and fuzzy sample entropy as the dependent variable was employed to estimate the difference between conscious sedation and monitored anesthesia care. In case of significant difference, post hoc t-tests were employed between PRE and POST and between conscious sedation and monitored anesthesia care.

The PRE and POST fuzzy sample entropy scores were used to construct receiver operating characteristic curves, and the area under these curves (AUC) [10] was computed by trapezoidal integration. The 95\% confidence intervals for AUC were estimated by bootstrap analysis (MATLAB bootci routine using the bias corrected accelerated percentile method) utilizing 1000 samples. Higher AUC (from 0.5 to 1) indicates a more distinct separation between PRE and POST.

\section{Results}

Patient characteristic are summarized in Table 1. There were no significant differences in age, height, weight, sex, or time from PRE to POST measurement. Sedation in conscious sedation consisted of midazolam $63 \pm 22 \mu \mathrm{g} / \mathrm{kg}$ and fentanyl $1.5 \pm 0.6 \mu \mathrm{g} / \mathrm{kg}$. In 16 patients, benadryl $0.49 \pm 0.18 \mathrm{mg} / \mathrm{kg}$ was employed due to inadequate sedation with the primary agents; excluding these patients did not alter the results. Sedation in monitored anesthesia care patients consisted of propofol $3.7 \pm 2.0 \mathrm{mg} / \mathrm{kg}$ and fentanyl $0.49 \pm 0.43 \mu \mathrm{g} / \mathrm{kg}$.

The mixed-design ANOVA showed a significant main effect between conscious sedation and monitored anesthesia care sedation groups $(p<0.001)$, between PRE and POST $(p<0.0001)$, and between eyes open and eyes closed $(p<0.0001)$. Post hoc tests found no difference between groups in PRE condition (both eyes open and eyes closed), but revealed a significant difference $(p<0.0001)$ between sedation groups in POST condition (both eyes open and eyes closed). Significant interaction was found between PRE/POST and sedation groups $(p<0.0001)$. Fuzzy sample entropy and post hoc results are presented in Table 2.

Receiver operating characteristic curves are presented in Figure 1.

Table 1. Patient characteristics

\begin{tabular}{|l|c|c|}
\hline & Conscious sedation & Monitored anesthesia care \\
\hline Age $($ years $)$ & $57.1 \pm 12.1$ & $55.8 \pm 14.7 \mathrm{NS}$ \\
\hline Height $(\mathrm{cm})$ & $168.5 \pm 9.9$ & $169.4 \pm 10.5 \mathrm{NS}$ \\
\hline Weight $(\mathrm{kg})$ & $82.7 \pm 18.9$ & $82.3 \pm 19.3 \mathrm{NS}$ \\
\hline Sex $(\mathrm{M} / \mathrm{F})$ & $42 / 61$ & $41 / 51 \mathrm{NS}$ \\
\hline Time from PRE to POST (hours) & $2.2 \pm 0.7$ & $2.3 \pm 0.6 \mathrm{NS}$ \\
\hline Midazolam $(\mu \mathrm{g} / \mathrm{kg})$ & $63 \pm 22$ & - \\
\hline Propofol $(\mathrm{mg} / \mathrm{kg})$ & - & $3.7 \pm 2.0$ \\
\hline Fentanyl $(\mu \mathrm{g} / \mathrm{kg})$ & $1.5 \pm 0.6$ & $0.49 \pm 0.43$ \\
\hline Benadryl $(\mathrm{mg} / \mathrm{kg})$ & $0.49 \pm 0.18$ & - \\
\hline
\end{tabular}

NS: Not Significant

Table 2. Mean and standard deviation of fuzzy sample entropy before (PRE) and after (POST) sedation in eyes open and eyes closed conditions.

\begin{tabular}{|l|c|c|c|c|}
\hline & \multicolumn{2}{|c|}{ Eyes open } & \multicolumn{2}{c|}{ Eyes closed } \\
\hline & CS & MAC & CS & MAC \\
\hline PRE & $0.31 \pm 0.05^{* *}$ & $0.30 \pm 0.05^{* * *}$ & $0.33 \pm 0.05$ & $0.34 \pm 0.06$ \\
\hline POST & $0.25 \pm 0.05^{* * *,+\dagger \dagger}$ & $0.29 \pm 0.06^{* * *}$ & $0.27 \pm 0.06^{+\dagger \dagger}$ & $0.32 \pm 0.06$ \\
\hline AUC & $0.83^{+++}$ & $0.56^{+}$ & $0.80^{+++}$ & $0.60^{+++}$ \\
\hline $95 \%$ CI of AUC & $0.78 \ldots 0.87$ & $0.46 \ldots 0.62$ & $0.76 \ldots 0.84$ & $0.55 \ldots 0.66$ \\
\hline
\end{tabular}

AUC is the area under receiver operating curve between PRE and POST. ${ }^{+}$indicates $p<0.05,{ }^{+++}$indicates $p<0.0001$ between PRE and POST. "indicates $p<0.001$ and ${ }^{* * *}$ indicates $p<0.0001$ between eyes open and eyes closed. ${ }^{\dagger \dagger}$ indicates $p<0.0001$ between conscious sedation (CS) and monitored anesthesia care (MAC). 


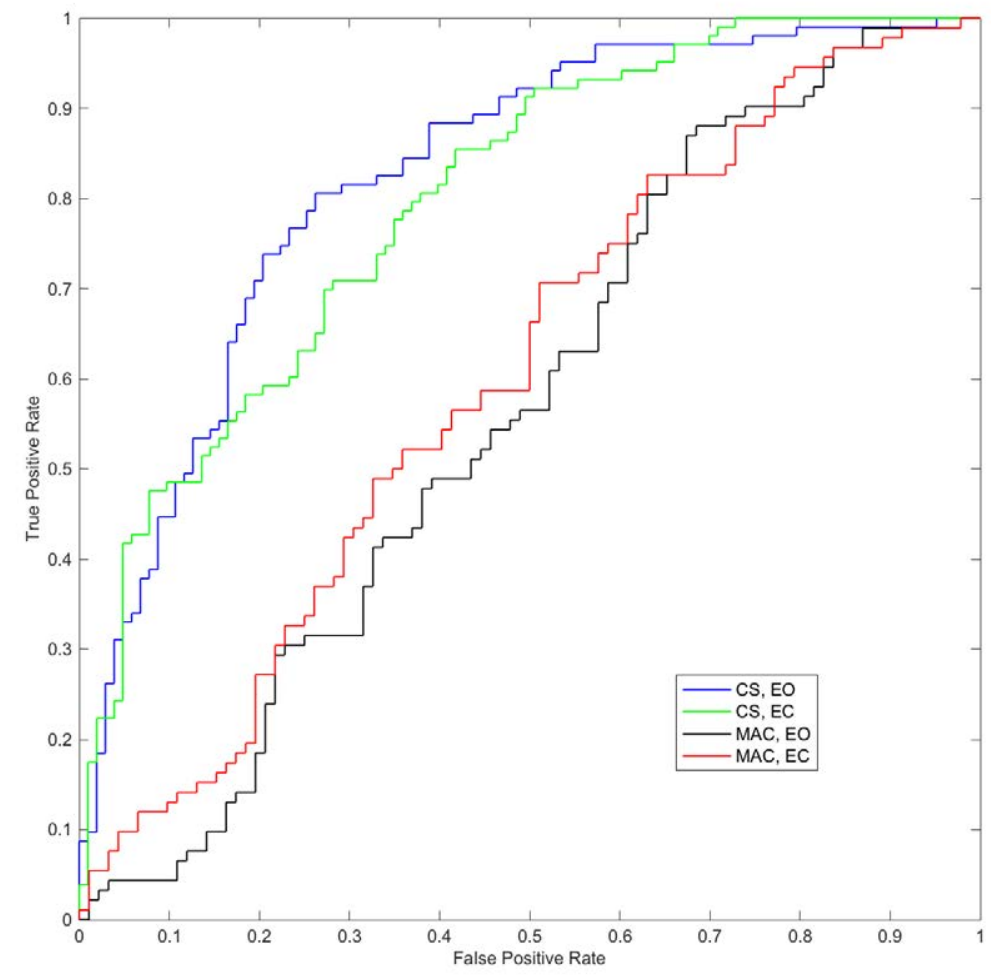

Figure 1. Receiver operating characteristic curves for conscious sedation (CS) and monitored anesthesia care (MAC) in eyes open (EO) and eyes closed (EC) condition.

The area under curve (AUC) for conscious sedation was 0.83 for eyes open and 0.80 for eyes closed, while for monitored anesthesia care, AUC was 0.56 for eyes open and 0.60 for eyes closed. The results of the post hoc tests between PRE and POST conditions are presented in Table 2.

\section{Discussion}

The major result of this study is the demonstration that in two patient groups whose principal difference was the sedative regimen, differentiation of the PRE and POST state by fuzzy sample entropy was significantly lower in the group who received a sedative less likely to produce residual postural impairment. This result suggests that fuzzy sample entropy of postural sway measures the effect of sedatives on postural control, rather than factors associated with endoscopy, such as bloating due to retained insufflating gas, or a learning factor for postural steadiness.

The significant differences between the main effect of conscious sedation vs. monitored anesthesia care $(p<0.001)$, the significant interaction between conscious sedation/monitored anesthesia care and PRE/POST $(p<0.0001)$, and the significant difference $(p<0.0001)$ between groups in POST condition (both eyes open and eyes closed), support the hypothesis that there is a difference in the patient's post-sedative steadiness between conscious sedation and monitored anesthesia care. The significant $(p<0.0001)$ main effect in PRE/POST, and in eyes open/eyes closed suggests that PRE and POST, as well as eyes open and eyes closed are different. While this is interesting in terms of understanding the effect of various sedative agents on postural control, either eyes open or eyes closed data is useable for detecting recovery, and eyes closed places patients at greater risk of falling.

Fuzzy sample entropy decreased in conscious sedation with both eyes open (AUC $=0.83, p<0.0001)$ and eyes closed $(\mathrm{AUC}=0.80$, $p<0.0001)$. The decrease in fuzzy sample entropy after monitored anesthesia care was smaller: $\mathrm{AUC}=0.56, p<0.05$ with eyes open and AUC $=0.60, p<0.0001$ with eyes closed. The decrease in the patients' postural steadiness (evidenced as an increase in sway regularity) has been associated with increased cognitive involvement in maintaining stable posture [11]. We previously demonstrated that this is also true when maintaining sedation impaired balance [8] and sleep-deprived balance [12]. The results in the current study are in agreement with previous findings that propofol impairs postural steadiness, but less so than midazolam $[3,4]$.

Since postural steadiness is affected by many factors, it is reasonable to ask whether the effects of endoscopy, such as residual insufflating gas, could explain persistent decreases in fuzzy sample entropy in POST condition. Some change may even come from merely repeating the measurements (i.e. "learning effect" [13]). While the results cannot exclude these as contributing factors, if this effect were solely responsible for the decrement in fuzzy sample entropy in monitored anesthesia care, it would constitute only a small portion of the effect in conscious sedation. While we have found that with practice it is possible to employ behaviors that increase or decrease the fuzzy sample entropy, it is unlikely that patients employed a deliberate strategy to bias the experimental results.

An advantage of the described approach is the simplicity. The Nintendo Wii Balance Board is an inexpensive device that is readily transported to the bedside, poses no electrical safety hazards, and can be used without a safety harness. The measurements take under 3 minutes for both eyes open and eyes closed, and do not require a physician or nurse to perform. While several patients required assistance to avoid falling with eyes closed, if only the eye open component of the test is employed, the likelihood of falling is negligible. 
There are several limitations of this study. First, patients were not randomized to treatment groups, and while there was no significant difference between patients' anthropometrics or in fuzzy sample entropy in the PRE condition (either with eyes open or eyes closed), the monitored anesthesia care group included both patients who had failed prior attempts at conscious sedation and patients who were judged by their gastroenterologists to be at risk for failure of conscious sedation. While it is likely that had patients who arrived for their endoscopy with expectation of conscious sedation been offered the possibility of monitored anesthesia care as a condition of participation in the study (as was done in our previous study [9]) a lower dose of propofol might have been required, it is unlikely that this would have reduced the observed effect, as sedation was titrated to tolerance of endoscopy. A second limitation is that while patients receiving monitored anesthesia care are more likely to meet ambulation criteria well in advance of discharge, we made no attempt to correlate the time to fitness for ambulation with fuzzy sample entropy during the POST period. We do not know whether propofol has less of an effect on postural steadiness than midazolam, or an equal effect that resolves more quickly, only that when meeting our institution's criteria for recovery room discharge, patients receiving conscious sedation were far more likely to be different from PRE.

Although there is no data to support the contention that patients with demonstrable alteration in postural steadiness have a higher rate of injury than those who have returned to baseline, we observed that patients receiving midazolam frequently required an escort to support them when leaving the endoscopy center, whereas this was rarely the case with propofol. Our results suggest that fuzzy sample entropy of postural sway is a simple screening method that can discriminate two patient groups that can reasonably be expected to have differences in street readiness. Further study is required to determine whether patients returning to postural steadiness prior to recovery room discharge can more rapidly return to activities of daily living, and whether failure to recover to baseline steadiness places patients at risk for falls.

\section{References}

1. Fujisawa T, Takuma S, Koseki H, Kimura K, Fukushima K. (2005) Assessment of the recovery of dynamic balance after intravenous sedation with midazolam. $J$ Anesth 19 : 26-30. [Crossref]

2. Fujisawa T, Takuma S, Koseki H, Kimura K, Fukushima K (2006) Recovery of intentional dynamic balance function after intravenous sedation with midazolam in young and elderly subjects. Eur J Anaesthesiol 23: 422-425. [Crossref]

3. Fujisawa T, Takuma S, Koseki H, Kimura K, Fukushima K (2007) Study on the usefulness of precise and simple dynamic balance tests for the evaluation of recovery from intravenous sedation with midazolam and propofol. Eur J Anaesthesiol 24: 425430. [Crossref]

4. Fujisawa T, Takuma S, Koseki H, Kimura K, Fukushima K (2007) Correlation between precise and simple tests in recovery of dynamic balance function after intravenous sedation with midazolam in the elderly. J Anesth 21: 153-158. [Crossref]

5. Lipsitz LA (2002) Dynamics of stability: the physiologic basis of functional health and frailty. J Gerontol A Biol Sci Med Sci 57: B115-125. [Crossref]

6. Caballero C, Barbado D, Moreno FJ (2015) What COP and Kinematic Parameters Better Characterize Postural Control in Standing Balance Tasks? J Motor Behavior 47 550-62. [Crossref]

7. Turnock MJ, Layne CS (2010) Variations in linear and nonlinear postural measurements under achilles tendon vibration and unstable support-surface conditions. J Motor Behav 42: 61-69. [Crossref]

8. Tietäväinen A, Hæggström E, Mandel JE (2014) Evaluation of postural steadiness before and after sedation: comparison of four nonlinear and three conventional measures. Physiol Meas 35: 139-151. [Crossref]

9. Mandel JE, Tanner JW, Lichtenstein GR, Metz DC, Katzka DA, et al. (2008) A randomized, controlled, double-blind trial of patient-controlled sedation with propofol/ remifentanil versus midazolam/fentanyl for colonoscopy, Anesth Analg 106: 434-439. [Crossref]

10. Zweig MH, Campbell G (1993) Receiver-operating characteristic (ROC) plots: a fundamental evaluation tool in clinical medicine. Clin Chem 39: 561-77. [Crossref]

11. Donker SF, Roerdink M, Greven AJ, Beek PJ (2007) Regularity of center-of-pressure trajectories depends on the amount of attention invested in postural control. Exp Brain Res 181: 1-11. [Crossref]

12. Tietäväinen A, Gates FK, Meriläinen A, Mandel JE, Hæggström E (2013) Nintendo $\mathbb{R}$ Wii Fit based sleepiness tester detects impairment of postural steadiness due to $24 \mathrm{~h}$ of wakefulness. Med Eng Phy 35: 1850-1853. [Crossref]

13. Wrisley DM, Stephens MJ, Mosley S, Wojnowski A, Duffy J, et al. (2007) Learning effects of repetitive administrations of the sensory organization test in healthy young adults. Arch Phys Med Rehabil 88: 1049-1054. [Crossref]

Copyright: (C2017 Mandel JE. This is an open-access article distributed under the terms of the Creative Commons Attribution License, which permits unrestricted use, distribution, and reproduction in any medium, provided the original author and source are credited. 